\title{
Efficacy of treatment for hyperglycemic crisis in elderly diabetic patients in a day hospital
}

\author{
D Benaiges ${ }^{1-3}$ \\ JJ Chillarón ${ }^{1-3}$ \\ MJ Carrera ${ }^{1,3}$ \\ F Cots ${ }^{3,4}$ \\ J Puig de Dou' \\ E Corominas' \\ J Pedro-Botet ${ }^{1-3}$
}

JA Flores-Le Roux ${ }^{1-3}$

C Claret'

A Goday ${ }^{1-3}$

JF Cano ${ }^{1-3}$

'Department of Endocrinology and Nutrition, Hospital del Mar, ${ }^{2}$ Department of Medicine, Universitat Autònoma de Barcelona, ${ }^{3}$ Institut Hospital del Mar d'Investigacions Mèdiques, ${ }^{4}$ Epidemiology and Evaluation Department, Parc de Salut Mar, Barcelona, Spain
Correspondence: $\mathrm{D}$ Benaiges Department of Endocrinology and Nutrition, Hospital del Mar, Passeig Marítim 25-29, E-08003 Barcelona, Spain

Tel +34932483902

$\mathrm{Fax}+34932483254$

Email 96002@parcdesalutmar.cat
This article was published in the following Dove Press journal:

Clinical Interventions in Aging

14 May 2014

Number of times this article has been viewed

Background: The purpose of this prospective cohort study was to compare the costs of day hospital $(\mathrm{DH})$ care for hyperglycemic crisis in elderly diabetic patients with those of conventional hospitalization $(\mathrm{CH})$. Secondary objectives were to compare these two clinical scenarios in terms of glycemic control, number of emergency and outpatient visits, readmissions, hypoglycemic episodes, and nosocomial morbidity.

Methods: The study population comprised diabetic patients aged $>74$ years consecutively admitted to a tertiary teaching hospital in Spain for hyperglycemic crisis (sustained hyperglycemia [ $>300 \mathrm{mg} / \mathrm{dL}$ ] for at least 3 days with or without ketosis). The patients were assigned to $\mathrm{DH}$ or $\mathrm{CH}$ care according to time of admission and were followed for 6 months after discharge. Exclusion criteria were ketoacidosis, hyperosmolar crisis, hemodynamic instability, severe intercurrent illness, social deprivation, or Katz index $>$ D.

Results: Sixty-four diabetic patients on DH care and 36 on $\mathrm{CH}$ care were included, with no differences in baseline characteristics. The average cost per patient was $1,345.1 \pm 793.6 €$ in the DH group and 2,212.4 $\pm 982.5 €$ in the $\mathrm{CH}$ group $(P<0.001)$. There were no differences in number of subjects with mild hypoglycemia during follow-up (45.3\% DH versus $33.3 \% \mathrm{CH}, P=0.24)$, nor in the percentage of patients achieving a glycated hemoglobin $\left(\mathrm{HbA}_{1 \mathrm{c}}\right)<8 \%(67.2 \% \mathrm{DH}$ versus $58.3 \% \mathrm{CH}, P=0.375)$. Readmissions for hyperglycemic crisis and pressure ulcer rates were significantly higher in the $\mathrm{CH}$ group.

Conclusion: DH care for hyperglycemic crises is more cost-effective than $\mathrm{CH}$ care, with a net saving of 1,418.4 $€$ per case, lower number of readmissions and pressure ulcer rates, and similar short-term glycemic control and hypoglycemia rates.

Keywords: day hospital, conventional hospitalization, hyperglycemic crisis

\section{Introduction}

Diabetes mellitus is a chronic disease with a major socioeconomic impact. In Spain, diabetes-related costs were estimated to be 8,509 million Euros in 2012, representing $8.2 \%$ of the national health expenditure. ${ }^{1}$

The prevalence of diabetes in Spain in 2010 was estimated to be $13.8 \%$, and this figure increases progressively with age. In this respect, 20.7\% of Spanish men over the age of 75 years have a diagnosis of diabetes and $16.7 \%$ have undiagnosed diabetes. $^{2}$ In addition, diabetes in the elderly is associated with a higher prevalence of other cardiovascular risk factors, coronary heart disease, stroke, premature death, physical disability, and geriatric syndromes..$^{3-9}$ These data could explain why the population aged over 65 years is responsible for $56 \%$ of health expenditure on diabetes. ${ }^{10}$

Hospital admissions have a huge impact on the costs of diabetes to the health system, ${ }^{11-13}$ and in Spain they represent one third of the total cost. ${ }^{1}$ In this respect, hyperglycemic crises are responsible for $7.1 \%$ of the hospital costs for diabetes, accounting 
for 70.7 million Euros in 2012. Hyperglycemic crises in elderly subjects have traditionally required conventional hospitalization $(\mathrm{CH})$ for their management. The day hospital (DH) is a consolidated form of hospitalization which avoids the drawbacks of $\mathrm{CH}$ and has social, psychological, medical, and economic advantages. $\mathrm{DH}$ is beneficial in several disorders where patients continue to have good physical activity and where medical health education and lifestyle play an important role. In this regard, $\mathrm{DH}$ has been demonstrated to be effective in the treatment of hyperglycemic crises in younger diabetics ${ }^{14,15}$ and in the management of diseases of the elderly, such as Parkinson's disease, Alzheimer's disease, and certain psychiatric disorders. ${ }^{16-18}$

The primary aim of this study was to compare the treatment costs of hyperglycemic crises when managed by $\mathrm{DH}$ and $\mathrm{CH}$ in diabetic subjects $>74$ years of age. The secondary objectives were to compare the effectiveness of these two clinical scenarios in terms of glycemic control, emergency and outpatient visits, readmissions, rates of hypoglycemia, and in-hospital morbidity.

\section{Materials and methods}

A nonrandomized prospective cohort study of patients $>74$ years of age admitted for hyperglycemic crises with a minimum 6-month follow-up was conducted at the Hospital del Mar, Barcelona. The study was approved by the ethics committee of the Hospital del Mar.

\section{Inclusion and exclusion criteria}

Patients with sustained hyperglycemia ( $>300 \mathrm{mg} / \mathrm{dL}$ ) for at least 3 days with or without ketosis were included in the study. Exclusion criteria were ketoacidosis (venous $\mathrm{pH}<7.31$ and/or $\mathrm{HCO}_{3}<22 \mathrm{mEq}$ ), hyperosmolar crisis (glycemia $>600 \mathrm{mg} / \mathrm{dL}$ and effective plasma osmolarity $>320 \mathrm{mOsm} / \mathrm{L}$ ), unstable hemodynamic status or need for ventilatory support, severe precipitating factors such as acute myocardial infarction, stroke, sepsis, social deprivation, and dependence for four or more activities of daily living (Katz index $>D$ ).

\section{Group assignment}

Patients were assigned to the DH group if they were admitted to hospital within DH opening hours (week days from 8 am to $4 \mathrm{pm}$ ); otherwise they were treated in the emergency department and subsequently hospitalized. Patients were treated following the same protocol for both $\mathrm{DH}$ and $\mathrm{CH}$. This protocol included initial evaluation with a blood test, urinalysis, and a chest radiograph to rule out underlying infectious disease, and hourly measurement of glycemia and ketonemia. Treatment included hydration with intravenous saline solution as required, an insulin regimen with insulin aspart administered subcutaneously every 2 hours, and oral carbohydrate intake if glucose levels were less than $250 \mathrm{mg} / \mathrm{dL}$ with persistent ketosis. If an infection was diagnosed, appropriate treatment was initiated. Diabetes education was delivered by a specialist diabetes nurse and was the same for patients in both groups, with specific attention paid to dietary advice, physical activity, and recognition of hypoglycemia.

After initial treatment of hyperglycemic crisis (for up to 8 hours), DH patients were scheduled for follow-up visits at 24, 72 hours, and 7 days to adjust treatment and to complete their diabetes education. At discharge, $\mathrm{CH}$ patients were scheduled for a one-week follow-up visit in the outpatient clinic. Measurement of glycated hemoglobin $\left(\mathrm{HbA}_{1 \mathrm{c}}\right)$ and clinical evaluation was scheduled for 3 and 6 months for patients in both groups.

At the time of admission for hyperglycemic crisis, data on the following baseline characteristics were collected: age, sex, weight, height, $\mathrm{HbA}_{1 \mathrm{c}}$, presence of chronic microvascular or macrovascular complications, diabetes treatment, diabetes duration, Katz index score, dependence for activities of daily living, Charlson comorbidity index score, and degree of family support. At the 3-month follow-up, the number of mild and severe hypoglycemic episodes, readmissions for any diabetes-related or unrelated cause, nosocomial complications (hospital-acquired infections and pressure ulcers), and number of outpatient and emergency room visits were recorded.

\section{Definition of clinical outcomes}

"Failure of treatment in DH" was defined as the need for $\mathrm{CH}$ in the first week after a patient was admitted for treatment in DH. This failure could be due to persistent hyperglycemia, ketosis or acidosis, severe intercurrent illness, or repeated hypoglycemia. Costs of hospital admissions attributed to $\mathrm{DH}$ failure were included in the cost of $\mathrm{DH}$ and not as readmissions. Mild hypoglycemia was defined as capillary glycemia $<60 \mathrm{mg} / \mathrm{dL}$ associated with adrenergic symptoms. Following the Diabetes Control and Complications Trial criteria, severe hypoglycemia was considered to be requirement of assistance for recovery or glucagon injection. ${ }^{19}$

\section{Laboratory measurements}

Ketone bodies were measured by determination of hydroxybutyrate in a capillary blood sample using a ketone monitoring system (Precision Xtra ${ }^{\circledR}$; Abbott Laboratories, 
North Chicago, IL, USA) and by determination of acetoacetate in a urine sample using Ketostix ${ }^{\circledR}$ (Bayer Corporation, Elkhart, UK). $\mathrm{HbA}_{1 \mathrm{c}}$ was measured using a commercial chromatographic method (Biosystems, Barcelona, Spain), certified by the National Glycohemoglobin Standardization Program as having documented traceability to the Diabetes Control and Complication Trial reference method and standardized to National Glycohemoglobin Standardization Program units.

\section{Costs}

Cost calculation included: initial care, pharmacy, complementary examinations, outpatient care, and readmission. Cost calculations for initial care in $\mathrm{CH}$ included emergency room stay and subsequent hospitalization. Cost calculations for initial care in DH included the DH stay and the costs associated with failure of treatment in DH. Outpatient costs included outpatient visits and emergency attendances not requiring hospital admission.

The methodology of the EuroDRG (Diagnosis-Related Groups) project was used for the cost calculations. ${ }^{20}$ The hospital uses a cost accounting system based on full-costing allocation that allows for assessing direct costs derived from clinical activity. In the present study, cost estimation was based on a full-cost accounting system and on the criteria of clinical activity-based costing methods to obtain the highest sensitivity in the assessment of variability in clinical activity. Moreover, this system ensures that the hospital's total costs are distributed among the patients. Allocation was based on directly assigning the cost of the following services to the patient: laboratory, pharmacy, radiology, nuclear medicine, pathology, and prosthetics. The information systems contain exhaustive data on human resources and their activity, ie, storage, admissions planning, ambulatory and emergency care, operating rooms, diagnostic and complementary tests, and interhospital consultations. This information creates and automatically updates the cost drivers for overheads.

\section{Statistical analysis}

Accepting an alpha risk of 0.05 and a beta risk of 0.20 in a bilateral contrast, 36 patients would be required in each group to detect a difference of $1,000 €$ or more. The variability of cost, according to our institution's economic research department, was $1,423 €$. A loss to follow-up of $10 \%$ was assumed.

Data were expressed as the mean \pm standard deviation for continuous variables and as percentages and frequencies for categorical variables. The Student's $t$-test was performed to assess differences between two means. The chi-squared or Fisher's exact tests were used to evaluate the degree of association of categorical variables. A $P$-value $<0.05$ was considered to be statistically significant. The statistical analysis was done with Statistical Package for the Social Sciences version 12 software (SPSS Inc., Chicago, IL, USA).

\section{Results}

During the study period, a total of 100 diabetic patients aged 74 years and older were included, ie, 36 in the $\mathrm{CH}$ group and 64 in the DH group. The mean age was $80.4 \pm 4.8$ (75-95) years and 63\% of the patients were female. Ninety-eight patients had type 2 diabetes and two had corticosteroid-induced diabetes. With regard to type of hyperglycemic crisis, $64 \%$ had acute hyperglycemia without ketosis ( $>300 \mathrm{mg} / \mathrm{dL})$ and $36 \%$ presented with ketosis. The groups were comparable in terms of age, sex, body mass index, Katz index, and socioeconomic and diabetes characteristics. Biochemical parameters at the time of admission were also comparable (Table 1). Precipitating factors for hyperglycemic crisis were infection $(40 \%)$, nonadherence to treatment (14\%), corticosteroid therapy (12\%), new-onset diabetes (11\%), and other causes in the remaining 23\%. At discharge, $73.5 \%$ of patients were on neutral protamine Hagedorn or premixed insulin twice daily, $4.1 \%$ were on insulin glargine once daily, and $6.2 \%$ were on oral medications (metformin or sulfonylureas), with no differences between groups $(P=0.109)$.

\section{Clinical results}

A progressive reduction in $\mathrm{HbA}_{1 \mathrm{c}}$ levels was observed at 3-month and 6-month-follow-up, with no differences between groups (Figure 1). Most of the patients had reached an $\mathrm{HbA}_{1 \mathrm{c}}<8 \%$ at the end of follow-up (Figure 2). Only one patient in each group had severe hypoglycemia that occurred during the first 15 days. Twelve subjects in the $\mathrm{CH}$ group and 29 in $\mathrm{DH}$ group suffered at least one mild hypoglycemic episode during follow-up $(33.3 \%$ versus $45.3 \%, P=0.24$ ).

Average stay was 9.0 \pm 3.2 days in the $\mathrm{CH}$ group and a maximum of 8 hours in the DH group $(P<0.001)$. The number of outpatient visits was greater in the DH group given the protocol for this particular group. However, no difference was observed in the number of emergency department admissions. The number of readmissions for any cause at 3 months was higher in the $\mathrm{CH}$ group, but was not statistically significant. However, readmission rates for diabetes at 3 months were significantly higher in the $\mathrm{CH}$ group (Table 2). There were 
Table I Baseline characteristics

\begin{tabular}{|c|c|c|c|}
\hline & $\begin{array}{l}\text { Day hospital } \\
(n=64)\end{array}$ & $\begin{array}{l}\text { Conventional } \\
\text { hospitalization }(n=36)\end{array}$ & $P$-value \\
\hline Age (years) & $80.3 \pm 4.8$ & $80.6 \pm 4.6$ & 0.786 \\
\hline Female sex (\%) & $67.2 \%$ & $55.6 \%$ & 0.173 \\
\hline $\mathrm{BMI}\left(\mathrm{kg} / \mathrm{m}^{2}\right)$ & $26.1 \pm 4.9$ & $25.5 \pm 5.1$ & 0.665 \\
\hline Katz A or B index (\%) & 72.2 & 72.2 & 0.529 \\
\hline Charlson comorbidity index & $3.2 \pm 2.0$ & $3.3 \pm 1.7$ & 0.936 \\
\hline Family support (\%) & 88.1 & 97.1 & 0.136 \\
\hline Diabetes duration (years) & $14.4 \pm 8.0$ & $17.4 \pm 1 \mid .2$ & 0.220 \\
\hline Microvascular complications (\%) & 38.8 & 38.2 & 0.699 \\
\hline Macrovascular complications (\%) & 33.1 & 37.1 & 0.633 \\
\hline Insulin therapy (\%) & 30.4 & 22.9 & 0.298 \\
\hline $\mathrm{HbA}_{\mathrm{lc}}(\%)$ & $10.9 \pm 1.9$ & $10.7 \pm 2.1$ & 0.667 \\
\hline $\mathrm{HbA}$ & $96.1 \pm 21.0$ & $94.1 \pm 23.5$ & 0.667 \\
\hline Venous glucose (mg/dL) & $420.0 \pm 74.8$ & $451.0 \pm 98.6$ & 0.108 \\
\hline Venous $\mathrm{pH}$ & $7.39 \pm 0.06$ & $7.40 \pm 0.05$ & 0.404 \\
\hline Weight loss within previous 3 months $(\mathrm{kg})$ & $2.97 \pm 3.66$ & $1.4 \pm 2.8$ & 0.058 \\
\hline
\end{tabular}

Abbreviations: $\mathrm{BMI}$, body mass index; $\mathrm{HbA}_{1 \mathrm{c}}$, glycated hemoglobin.

three cases (4.6\%) of DH treatment failure, two cases due to persistent hyperglycemia, and one case in the context of nonadherence to treatment. With regard to nosocomial complications, five patients (13.8\%) in the $\mathrm{CH}$ group and none in the DH group developed superficial noninfected pressure ulcers $(P=0.002)$, and one was diagnosed with a nosocomial urinary tract infection.

\section{Financial cost results}

Although DH care generated more outpatient costs, the overall cost of $\mathrm{CH}$ was clearly higher (Table 3 ).

\section{Discussion}

DH care for diabetic patients with hyperglycemic crisis was an efficient practice, saving $1,418.4 €$ per patient. Moreover, there were no differences in glycemic control or

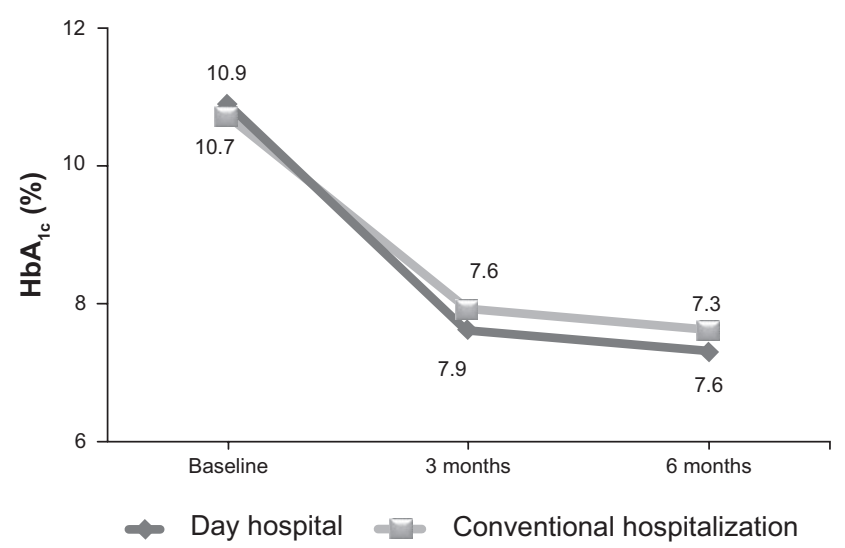

Figure I Glycated hemoglobin $\left(\mathrm{HbA}_{\mathrm{Ic}}\right)$ levels during follow-up. in the number of hypoglycemic events in the medium term, and DH patients did not suffer pressure ulcers and required less frequent diabetes-related readmission. This finding is important when considering the economic impact of diabetes on health systems. The annual health care expenditure associated with diabetes in the USA in 2007 was 174 billion dollars, of which 116 were associated with medical expenses and 58 with loss of labor hours. Further, the estimated annual cost associated with a case of diabetes in Spain is $1,770 € . .^{10}$ The economic benefit of DH care found in the present study is even more relevant if we take into consideration that elderly patients account for much of the health spending on diabetes. ${ }^{10}$

In the present study, DH care for elderly patients with hyperglycemic crisis avoided the costs associated with hospitalization, which accounted for approximately $85 \%$ of

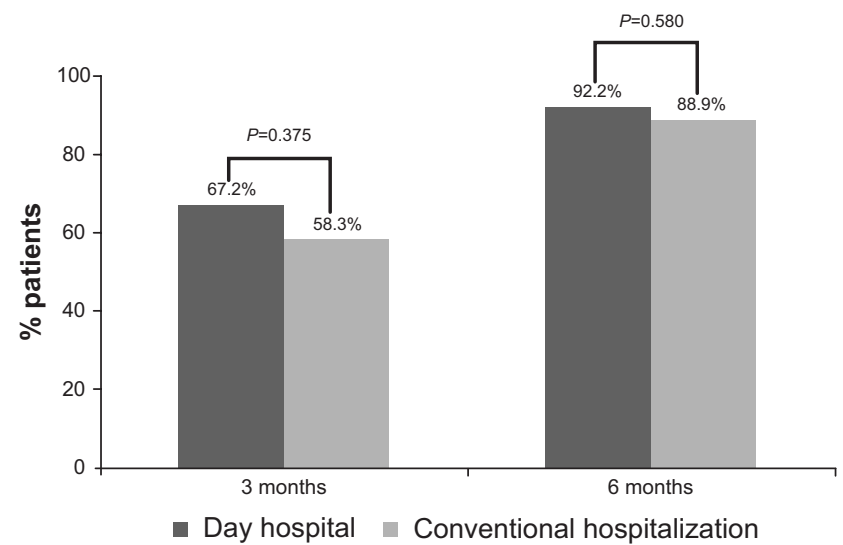

Figure 2 Percentage of patients achieving glycated hemoglobin $\left(\mathrm{HbA}_{\mathrm{lc}}\right)<8 \%$. 
Table 2 Study endpoints

\begin{tabular}{llll}
\hline & Day hospital & $\begin{array}{l}\text { Conventional } \\
\text { hospitalization }\end{array}$ & $P$-value \\
\hline Outpatient visits (n) & & $2.5 \pm 2.0$ & 0.012 \\
Emergency room admissions & $5.0 \pm 2.2$ & $0.2 \pm 0.4$ & 0.590 \\
Readmission for any cause (\%) & $0.2 \pm 0.6$ & $7(19.4)$ & 0.085 \\
Diabetes-related readmissions (\%) & $4(6.3)$ & $5(13.9)$ & 0.040 \\
\hline
\end{tabular}

the total cost in the $\mathrm{CH}$ group. It should be stressed that the cost of diabetes in the last year of life is mainly associated with a higher rate of hospitalization; moreover, the length of stay is $70 \%$ longer than in nondiabetic patients and it is associated with a greater number of complications, such as nosocomial infections, pressure ulcers, and adverse drug reactions. ${ }^{21}$

Despite a higher cost for outpatient visits ( $\mathrm{DH}$ care includes three follow-up visits in the first week) and three hospital admissions due to failure of DH care, DH is clearly beneficial when we compare the total costs of $\mathrm{CH}$.

In addition to the economic benefits, DH had further advantages over $\mathrm{CH}$. First, we observed a reduction in readmissions for diabetes. This may reflect the fact that patients treated by $\mathrm{CH}$ are living outside their usual environment and must adapt to a different routine, food intake, and physical activity. Thus, when patients are discharged, return to everyday habits may destabilize glycemic control and lead to readmission. Second, DH care prevented nosocomial complications, mainly the pressure ulcers that were present in approximately one in seven patients in the $\mathrm{CH}$ group. Third, other potential benefits not evaluated in the present study, but which may hypothetically favor DH care, are related to functional improvement, less emotional impact on the lives of the elderly, and a minor impact on the family.

Since the UK Prospective Diabetes Study showed an improvement in the risk of microvascular complications with intensive glycemic control, scientific societies have established the goal of $\mathrm{HbA}_{1 \mathrm{c}}<7 \%$ for all subjects with type 2 diabetes mellitus. ${ }^{22}$ The ACCORD (Action to Control Cardiovascular Risk in Diabetes), ${ }^{23}$ ADVANCE (Action in Diabetes and Vascular Disease: Preterax ${ }^{\circledR}$
[Servier International, Neuilly sur Seine, France] and Diamicron MR Controlled Evaluation), ${ }^{24}$ and VADT ${ }^{25}$ studies, published in 2009, designed for the diabetic population with an average age around 65 years and longstanding diabetes, showed that strict glycemic control had no effect on the risk of cardiovascular events, and was associated with increased mortality from all causes in the ACCORD study. ${ }^{23}$ This has led to a recommendation for less stringent control $\left(\mathrm{HbA}_{1 \mathrm{c}}\right.$ $<8 \%$ ) for patients with longstanding diabetes, fragility, and/ or cardiovascular disease, which represents the majority of elderly diabetic patients. ${ }^{26,27}$ In the present study, over $85 \%$ of patients in both groups achieved an average $\mathrm{HbA}_{1 \mathrm{c}}<8 \%$ at 6 months, and therefore adjusted to current goals.

Since the metabolic goal in this study was nonaggressive, ie, $\mathrm{HbA}_{1 \mathrm{c}}<8 \%$, there were few episodes of hypoglycemia in both groups. Nevertheless, it is important to emphasize that we found no difference in the number of hypoglycemic events between the groups. Hypoglycemia in the elderly can be associated with potentially serious consequences, ${ }^{28}$ so treatment of diabetes in this age group should aim to minimize this risk. ${ }^{29,30}$

Evidence-based guidelines for many health conditions that affect the elderly are deficient owing to the lack of studies in this age group; thus, the tendency is to extrapolate evidence from younger age groups. Several authors propose a change in patient care in the last years of life, advocating overall management rather than focusing on the different comorbidities. ${ }^{31,32}$ We consider DH care for hyperglycemic crisis to be in line with this new concept in the care of the elderly and their use could be extended to other chronic illnesses.

Table 3 Costs of treatment

\begin{tabular}{llll}
\hline & Day hospital & Conventional hospitalization & P-value \\
\hline Initial care $(€)$ & $580.2 \pm 489.1$ & $2,013.6 \pm 790.4$ & $<0.001$ \\
Complementary examinations $(€)$ & $123.7 \pm 276.3$ & $281.3 \pm 188.1$ & 0.007 \\
Pharmacy $(€)$ & $12.8 \pm 95.6$ & $20.3 \pm 24.8$ & 0.676 \\
Outpatient visits $(€)$ & $116.7 \pm 75.3$ & $56.9 \pm 105.7$ & 0.003 \\
Readmissions $(€)$ & $340.8 \pm 1190$ & $288.3 \pm 916.8$ & 0.835 \\
Total $(€)$ & $1,345.1 \pm 793.6$ & $2,212.4 \pm 982.5$ & $<0.001$ \\
\hline
\end{tabular}


The limitations of the present study are mainly related to the study design, given that patients were not randomly allocated to treatment groups. The method of assigning patients according to time of arrival at the emergency room could be a weakness: however, the bulk of hospital care is known to be concentrated during the day and less at night, ${ }^{33}$ and it is noteworthy that both groups had similar baseline characteristics. Moreover, severe hyperglycemic crisis was not included in the present study, so its results cannot be generalized to the treatment of these severe cases. We only estimated costs at the hospital level and other costs, such as use of social resources, and those arising from off-work relatives were not taken into account.

\section{Conclusion}

When compared with $\mathrm{CH}, \mathrm{DH}$ care for elderly diabetic patients with hyperglycemic crisis is an efficient practice in terms of expenditure, safety, and metabolic control.

\section{Acknowledgments}

This paper is published on behalf of the members of the DIABEMAR group, that includes D Benaiges, C Bosch, JF Cano, MJ Carrera, JJ Chillarón, C Claret, E Corominas, F Cots, B Dalama, M Fernández, JA Flores-Le Roux, A Goday, R Güerri, E Hernandez, A Mas, S Mauri, J Pedro-Botet, M Prados, J Puig de Dou, M Renard, and E Sagarra. This research was supported by the Spanish Ministry of Science and Innovation, the Health Research Fund (PI02/0401), and the Catalan Fundacio Agrupacio Mutua (Field of Aging Patients, 2005). We thank Miss Christine O'Hara for review of the English version of the manuscript.

\section{Disclosure}

The authors report no conflicts of interest in this work.

\section{References}

1. Crespo C, Brosa M, Lopez-Alba A, Lopez-Martinez N, Soria B. Direct cost of diabetes mellitus and its complications in Spain (SECCAID Study: Spain estimated cost Ciberdem-Cabimer in Diabetes). Av Diabetol. 2013;6:182-189. Spanish.

2. Soriguer F, Goday A, Bosch-Comas A, et al. Prevalence of diabetes mellitus and impaired glucose regulation in Spain: the Di@bet.es Study. Diabetologia. 2012;55:88-93.

3. Schwartz AV, Hillier TA, Sellmeyer DE, et al. Older women with diabetes have a higher risk of falls: a prospective study. Diabetes Care. 2002;25:1749-1754.

4. Songer TJ. Disability in diabetes. In: Harris MI, Cowie CC, Stern MP, editors. Diabetes in America. 2nd ed. Bethesda, MD, USA: National Institutes of Health; 1995.

5. Peyrot M, Rubin RR. Levels and risks of depression and anxiety symptomatology among diabetic adults. Diabetes Care. 1997;20: $585-590$.
6. Munshi M, Grande L, Hayes M, et al. Cognitive dysfunction is associated with poor diabetes control in older adults. Diabetes Care. 2006;29:1794-1799.

7. Brown JS, Seeley DG, Fong J, Black DM, Ensrud KE, Grady D. Urinary incontinence in older women. Who is at risk? Study of Osteoporotic Fractures Research Group. Obstet Gynecol. 1996;87:715-721.

8. Schwartz AV, Sellmeyer DE, Ensrud KE, et al; Study of Osteoporotic Features Research Group. Older women with diabetes have an increased risk of fracture: a prospective study. J Clin Endocrinol Metab. 2001;86:32-38.

9. Vinik AI. Diabetic neuropathy. Pathogenesis and therapy. Am J Med. 1999;107:17S-26S.

10. American Diabetes Association. Economic costs of diabetes in the US in 2007. Diabetes Care. 2008;31:596-615.

11. Donnan PT, Leese GP, Morris AD. Hospitalizations for people with type 1 and type 2 diabetes compared with the nondiabetic population of Tayside, Scotland: a retrospective cohort study of resource use. Diabetes Care. 2000;23:1774-1779.

12. Ray NF, Thamer M, Taylor T, Fehrenbach SN, Ratner R. Hospitalization and expenditures for the treatment of general medical conditions among the US diabetic population in 1991. J Clin Endocrinol Metab. 1996;81:3671-3679.

13. Aro S, Koivisto VA, Reunanen A, Salinto M, Kangas T. Influence of morbidity and health care structure on hospitalization among adult diabetic patients. Diabet Med. 1996;13:376-381.

14. Clar C, Waugh N, Thomas S. Routine hospital admission versus outpatient or home care in children at diagnosis of type 1 diabetes mellitus. Cochrane Database Syst Rev. 2007;2:CD004099.

15. Zghal A, el Fehik N, Bousnina O, Daoud I, Zghal I, Gaigi S. Diabetes and the day hospital. Tunis Med. 2000;78:223-226.

16. Marshall M, Crowther R, Almaraz-Serrano A, et al. Systematic reviews of the effectiveness of day care for people with severe mental disorders: (1) acute day hospital versus admission; (2) vocational rehabilitation; (3) day hospital versus outpatient care. Health Technol Assess. 2001;5:1-75.

17. Lai CK, Yeung JH, Mok V, Chi I. Special care units for dementia individuals with behavioural problems. Cochrane Database Syst Rev. 2009;2:CD006470.

18. Housley M. Educating the elderly diabetic at a day hospital. Prof Care Mother Child. 1994;4:142-144.

19. [No authors listed]. The effect of intensive treatment of diabetes on the development and progression of long-term complications in insulindependent diabetes mellitus. The Diabetes Control and Complications Trial Research Group: N Engl J Med. 1993;329:977-986.

20. Tan S, Serdén L, Geissler A, et al. DRGs and cost accounting: which is driving which. In: Busse R, Geissler A, Quentin W, Wiley M, editors. Diagnosis-Related Groups in Europe. Moving Towards Transparency, Efficiency and Quality in Hospitals. Maidenhead, UK: Open University Press; 2011.

21. Walter LC, Brand RJ, Counsell SR, et al. Development and validation of a prognostic index for 1-year mortality in older adults after hospitalization. JAMA. 2001;285:2987-2994.

22. [No authors listed]. Effect of intensive blood-glucose control with metformin on complications in overweight patients with type 2 diabetes (UKPDS 34). UK Prospective Diabetes Study (UKPDS) Group. Lancet. 1998;352:854-865.

23. Cushman WC, Evans GW, Byington RP, et al. Effects of intensive blood-pressure control in type 2 diabetes mellitus. N Engl J Med. 2010; 362:1575-1585.

24. ADVANCE Collaborative Group; Patel A, MacMahon S, Chalmers J, et al. Intensive blood glucose control and vascular outcomes in patients with type 2 diabetes. $N$ Engl J Med. 2008;358:2560-2572.

25. Duckworth W, Abraira C, Moritz T, et al. Glucose control and vascular complications in veterans with type 2 diabetes. $N$ Engl J Med. 2009;360:129-139.

26. American Diabetes Association. Standards of medical care in diabetes 2013. Diabetes Care. 2014;37:S14-S80. 
27. Sinclair A, Morley JE, Rodriguez-Mañas L, et al. Diabetes mellitus in older people: position statement on behalf of the International Association of Gerontology and Geriatrics (IAGG), the European Diabetes Working Party for Older People (EDWPOP), and the International Task Force of Experts in Diabetes. J Am Med Dir Assoc. 2012;13: 497-502.

28. Bremer JP, Jauch-Chara K, Hallschmid M, Schmid S, Schultes B. Hypoglycemia unawareness in older compared with middle-aged patients with type 2 diabetes. Diabetes Care. 2009;32:1513-1517.

29. Bruce DG, Davis WA, Casey GP, et al. Severe hypoglycaemia and cognitive impairment in older patients with diabetes: the Fremantle Diabetes Study. Diabetologia. 2009;52:1808-1815.
30. Chelliah A, Burge MR. Hypoglycaemia in elderly patients with diabetes mellitus: causes and strategies for prevention. Drugs Aging. 2004; 21:511-530.

31. Reuben DB. Medical care for the final years of life. "When you're 83, it's not going to be 20 years". JAMA. 2009;302:2686-2694.

32. Gómez Huelgas R, Díez-Espino J, Formiga F, et al; en nombre del Grupo de Trabajo para el Documento de Consenso sobre el tratamiento de la diabetes tipo 2 en el anciano. [Treatment of type 2 diabetes in the elderly]. Med Clin (Barc). 2013;140:1-134. Spanish.

33. Alonso M, Hernández R, Busto F, Cueto A. Utilización de un servicio de urgenciashospitalario. Rev Sanid Hig Publica (Madr). 1993; $67: 39-45$.
Clinical Interventions in Aging

\section{Publish your work in this journal}

Clinical Interventions in Aging is an international, peer-reviewed journal focusing on evidence-based reports on the value or lack thereof of treatments intended to prevent or delay the onset of maladaptive correlates of aging in human beings. This journal is indexed on PubMed Central, MedLine,

\section{Dovepress}

CAS, Scopus and the Elsevier Bibliographic databases. The manuscript management system is completely online and includes a very quick and fair peer-review system, which is all easy to use. Visit http://www.dovepress. com/testimonials.php to read real quotes from published authors. 